\title{
The Effects of Radiation Induced Carbon Contamination on the Performance of an EUV Lithographic Optic
}

Anton Barty

Kenneth A. Goldberg

The Society of Photo-Optical Instrumentation Engineers(SPIE) Santa Clara, Ca February 24 - 27, 2003

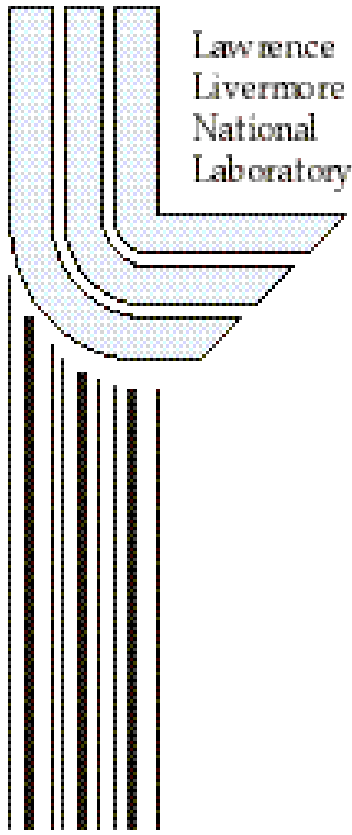

March 2003 


\section{DISCLAIMER}

This document was prepared as an account of work sponsored by an agency of the United States Government. Neither the United States Government nor the University of California nor any of their employees, makes any warranty, express or implied, or assumes any legal liability or responsibility for the accuracy, completeness, or usefulness of any information, apparatus, product, or process disclosed, or represents that its use would not infringe privately owned rights. Reference herein to any specific commercial product, process, or service by trade name, trademark, manufacturer, or otherwise, does not necessarily constitute or imply its endorsement, recommendation, or favoring by the United States Government or the University of California. The views and opinions of authors expressed herein do not necessarily state or reflect those of the United States Government or the University of California, and shall not be used for advertising or product endorsement purposes.

This is a preprint of a paper intended for publication in a journal or proceedings. Since changes may be made before publication, this preprint is made available with the understanding that it will not be cited or reproduced without the permission of the author.

This report has been reproduced directly from the best available copy.

Available electronically at http://www.doc.gov/bridge

Available for a processing fee to U.S. Department of Energy

And its contractors in paper from

U.S. Department of Energy

Office of Scientific and Technical Information

P.O. Box 62

Oak Ridge, TN 37831-0062

Telephone: (865) 576-8401

Facsimile: (865) 576-5728

E-mail: reports@adonis.osti.gov

Available for the sale to the public from

U.S. Department of Commerce

National Technical Information Service

5285 Port Royal Road

Springfield, VA 22161

Telephone: (800) 553-6847

Facsimile: (703) 605-6900

E-mail: orders@ntis.fedworld.gov

Online ordering: http:/ / www.ntis.gov/ordering.htm

\section{OR}

Lawrence Livermore National Laboratory

Technical Information Department's Digital Library

http:/ / www.llnl.gov/tid/Library.html 


\title{
The effects of radiation induced carbon contamination on the performance of an EUV lithographic optic
}

\author{
Anton Barty \\ Lawrence Livermore National Laboratory \\ PO Box 808, Livermore, CA, 94550 \\ Kenneth A. Goldberg \\ Centre for X-ray Optics, Lawrence Berkeley National Laboratory \\ Berkeley, CA, 94720
}

\begin{abstract}
Carbon deposition in EUVL is known to occur when optical surfaces in a hydrocarbon environment are exposed to EUV light. Carbon contamination on EUV optical elements affects both the absorption and phase of the reflected light. Because the carbon deposition alters the phase structure of the reflected EUV light it effectively alters the figure of these optics and, thus, the aberrations as well. Absorption by deposited carbon not only reduces throughput but also leads to apodisation of the pupil, which in turn affects imaging performance.
\end{abstract}

\section{Background}

Extreme ultraviolet lithography (EUVL) is one of the leading next-generation lithography technology. EUVL incorporates the use of all-reflective optics, including reflective masks. The Sandia 10x microstepper is a small-field multilayer coated Schwarzschild lithographic camera used primarily for EUV resist testing experiments. Operating at 10x demagnification with a field of view of $400 \mu \mathrm{m}$ and a numerical aperture of $0.08 \mathrm{NA}$ at the wafer, the typical spatial resolution is of the order of $100 \mathrm{~nm}$ at the wafer. An illustration of the system is shown in Figure 1.

Degradation in the performance of one of the 10x cameras was observed over time and attributed to the optical system, which was removed for analysis and realignment using the visible light interferometry. This visible light realignment and interferometric analysis indicated the presence of a 18-19nm bump in the centre of the optic which was not previously observed when the optic was first aligned. The system wavefront map as measured using visible light interferometry in November 2001 is shown below in Figure 2: on the left is the measured system wavefront at both visible and EUV wavelengths after initial assembly, and on the right is the system wavefront measured in November 2001 using visble light. 


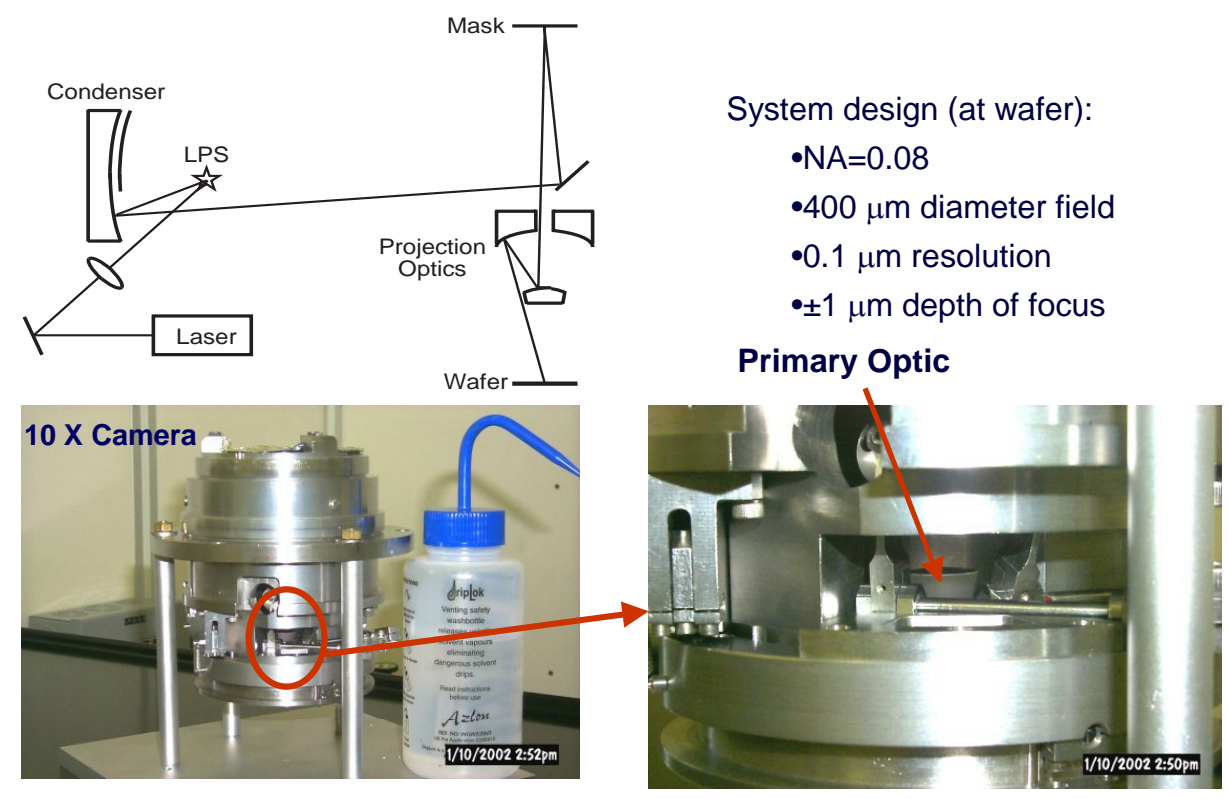

Figure 1

Schematic illustration of the 10x camera and optics assembly.
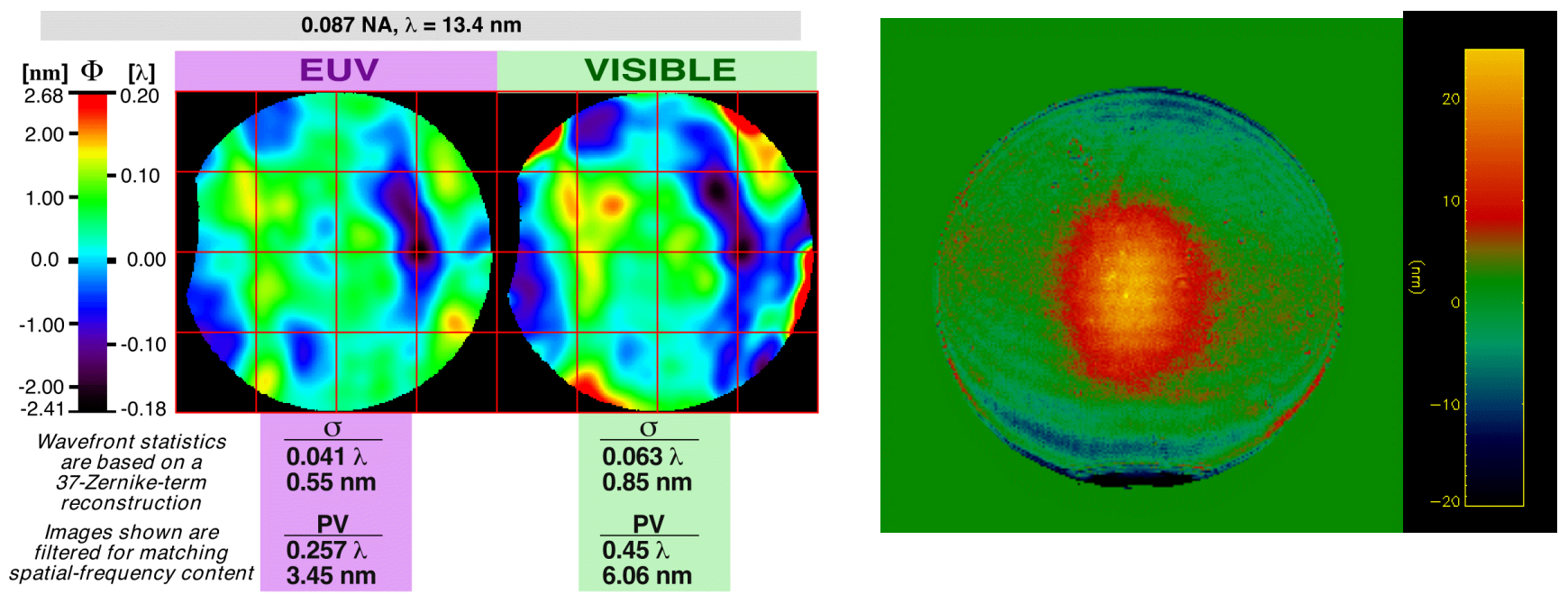

Figure 2

System wavefront of the 10x camera as measured using visible light interferometry after initial assembly (left) and in November 2001 after use in a comparatively hydrocarbon-rich environment (Рнс $10^{-7}$ torr).

$19 \mathrm{~nm}$ is a significant amount of wavefront error and would be expected to cause a significant amount of degradation in the lithographic performance of the optic. However, the observed EUV printing performance of the camera strongly indicated that the EUV performance of the camera was not as bad as one would expect from a $19 \mathrm{~nm}$ figure error on the optic. There was therefore a discrepancy between the measured system wavefront and the observed lithographic performance of this optic. The 10x camera had been used in a comparatively hydrocarbon rich environment, where the hydrocarbon partial pressure is of the order of $10^{-7}$ torr, thus accelerated carbon deposition is to be expected. One possible explanation for the difference in visible light and EUV performance is therefore the presence of carbon deposition on the surface of the optics: carbon is more transparent 
to EUV than to visible light so the effect of this bump on the wavefront would be less in the EUV region than in the visible.

\section{Reflected phase in the presence of carbon contamination}

The effect of carbon on a multilayer mirror can be modelled as effectively at visible wavelengths as at EUV and $\mathrm{x}$-ray wavelengths using standard multilayer calculation techniques. The electromagnetic equations are the same, as are key parameters characterising the multilayer such as bilayer period and number of layers. However, the values for the optical constants are quite different at the two different wavelengths.

To model the effects of carbon deposition on the multilayer optical performance we deposit a Gaussianshaped bump of carbon with the optical constants listed above and compute both the amplitude and phase of the reflected wave across the region of carbon deposition as shown in. For simplicity a multilayer of 50 bi-layer periods with no interface roughness and a $4 \mathrm{~nm}$ thick surface $\mathrm{SiO}_{2}$ oxide layer is assumed.

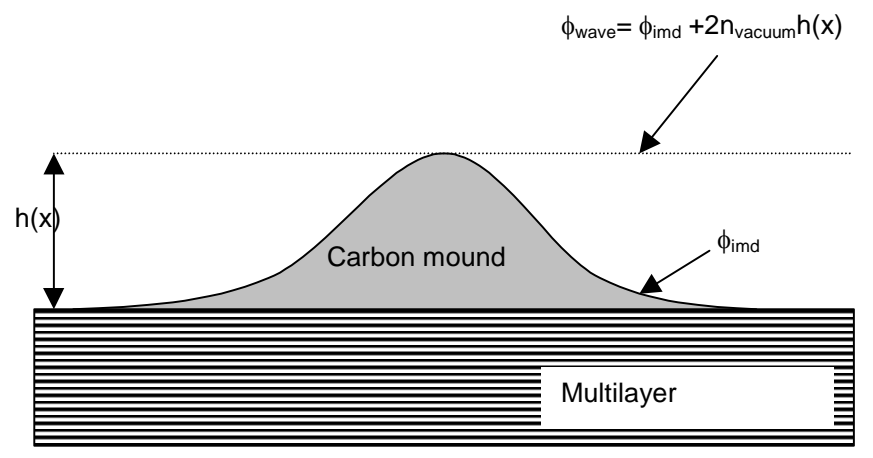

Figure 3

Carbon mound on an EUV multilayer used for modelling the effect

\begin{tabular}{|l|c|c|}
\hline \multicolumn{1}{|c|}{ Material } & $\begin{array}{c}\text { Visible light } \\
(533 \mathrm{~nm})\end{array}$ & $\begin{array}{c}\text { EUV } \\
(13.5 \mathrm{~nm})\end{array}$ \\
\hline Silicon & $4.177+0.052 \mathrm{i}$ & $0.998+0.00173 \mathrm{i}$ \\
\hline Molybdenum & $3.7+3.6 \mathrm{i}$ & $0.918+0.00734 \mathrm{i}$ \\
\hline Carbon & $2.3+0.87 \mathrm{i}$ & $0.961+0.00693 \mathrm{i}$ \\
\hline
\end{tabular}

Table 1

Pertinent optical constant values at visible and EUV wavelengths.

By calculating the reflected phase as a function of carbon thickness $h(x)$ at visible wavelengths (533nm) across the region of carbon deposition it is possible to deduce the carbon layer thickness $h(x)$ required to produce the additional figure error measured in the optic. Fitting the reflected phase to the observed figure error we quickly find that a $29 \mathrm{~nm}$ thick deposit of graphitic carbon produces a $38 \mathrm{~nm}$ phase shift in the reflected light, equivalent to the observed $19 \mathrm{~nm}$ figure error measured using $533 \mathrm{~nm}$ visible light, Figure 4 . Performing the same calculation at EUV wavelengths, however, yields a figure error of only $1.3 \mathrm{~nm}$, a difference which is wholly attributable to the difference in optical constants between the two wavelengths: Figure 5. 

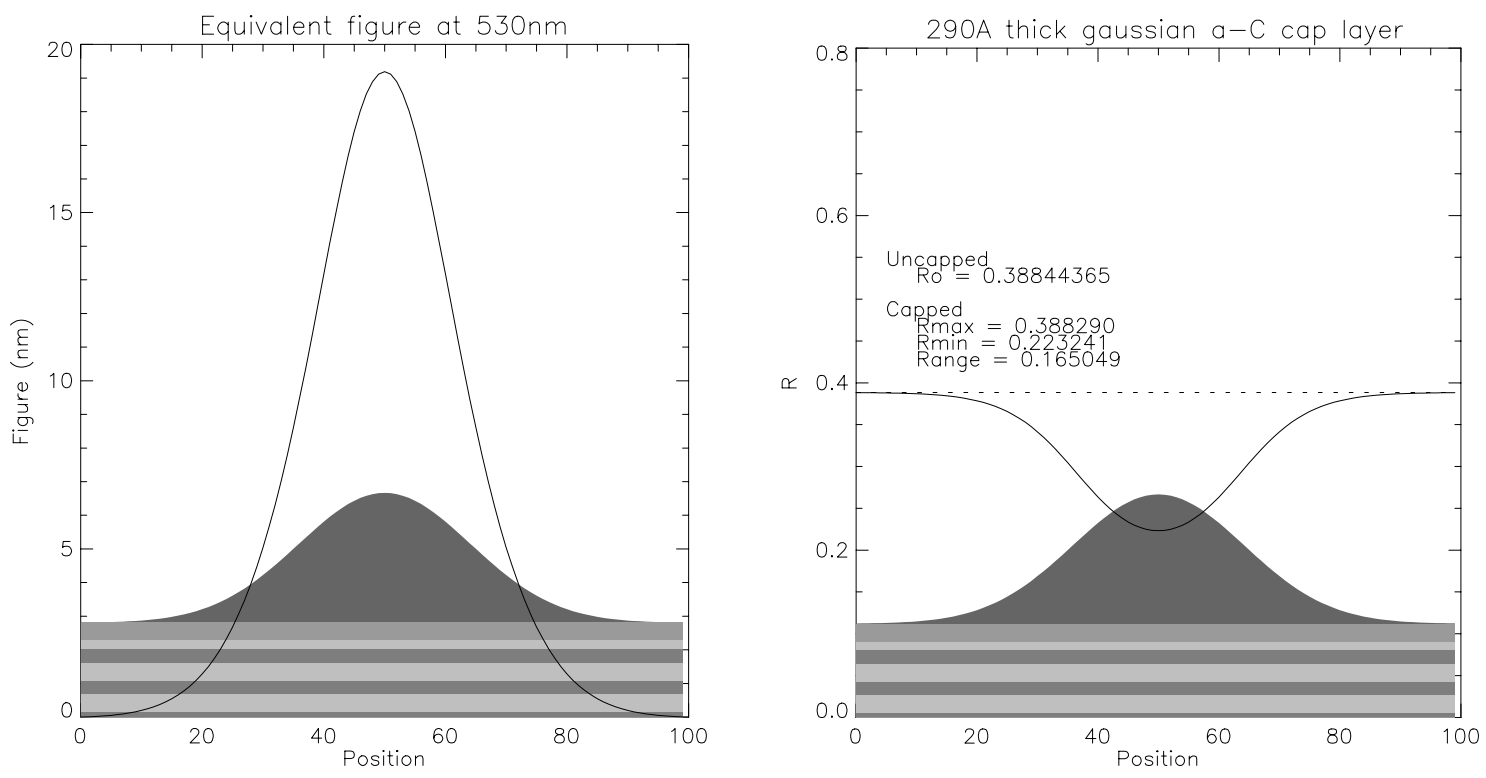

Figure 4

Calculated figure error (left) and reflectivity (right) at visible light wavelengths (533nm) caused by a 29nm thick Gaussianshaped carbon layer deposited on the surface on an EUV multilayer.
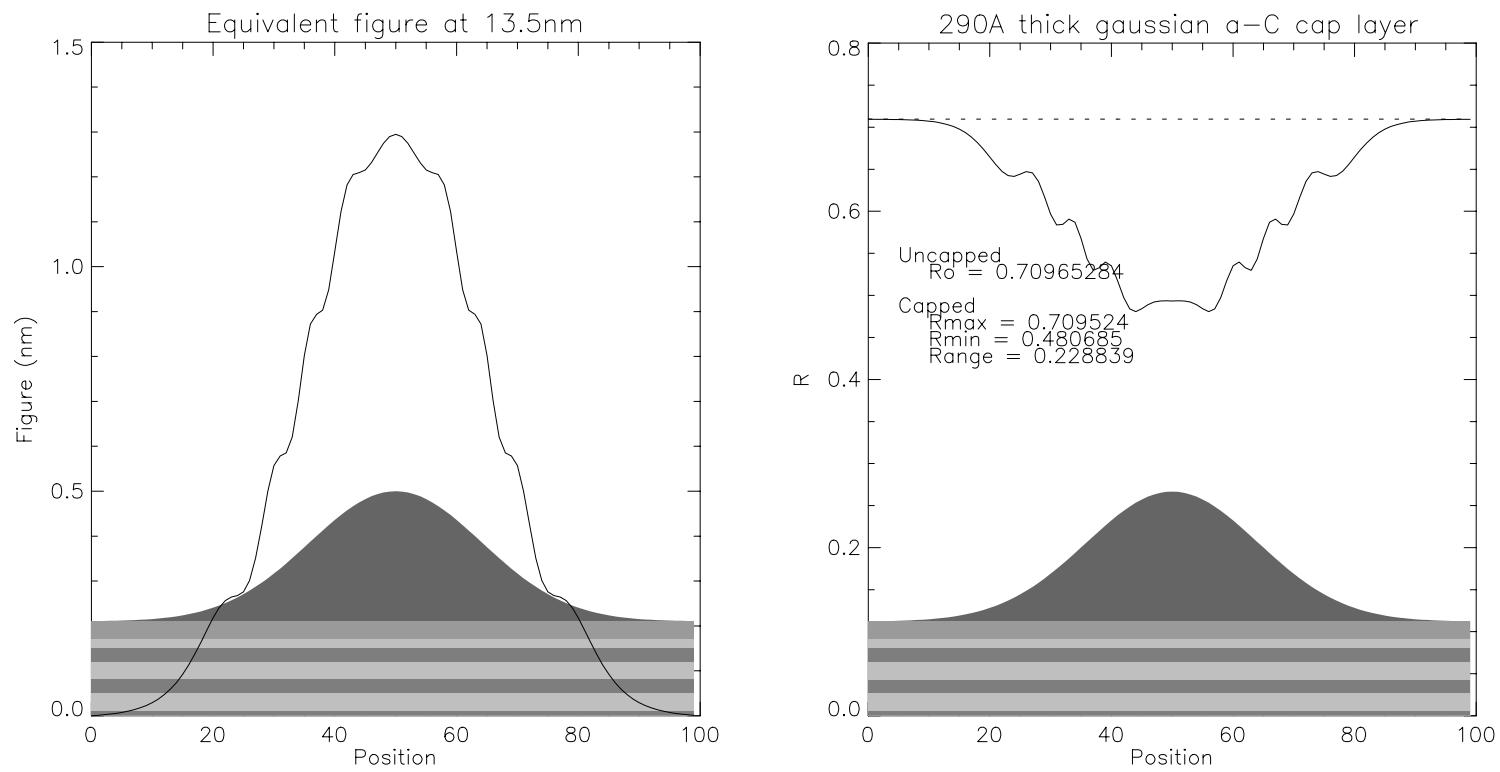

Figure 5

Calculated figure error (left) and reflectivity (right) at EUV wavelengths (13.5nm) caused by the same 29nm thick Gaussianshaped carbon layer depicted in Figure 4.

\section{Analysis of measured figure data}

There is sufficient data in the above analysis to convert the measured visible light interferometry data to predictions about the figure and reflectance of the 10x optic at EUV wavelengths. For the sake of simplicity we attribute all of the observed difference in measured figure to the presence of carbon on the surface. The figure 
degradation mechanism is assumed to be due to carbon deposition, deposited in the form of bulk amorphous material. Applying this strategy to the measured figure data, and assuming that carbon deposition is the sole source of the observed figure error, we can convert the measured visible light figure data into a carbon thickness map, Figure 6; and given this carbon thickness map we can directly compute the predicted EUV figure and reflectance, Figure 7.
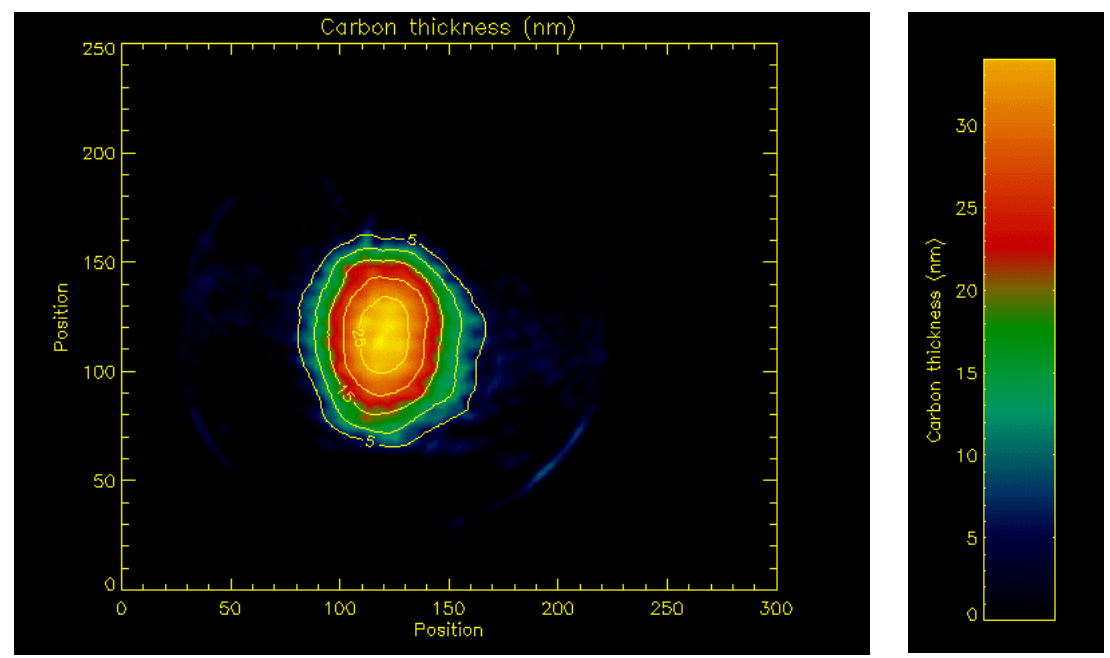

Carbon layer thickness calculated from measured figure error

Figure 6

Calculated carbon layer thickness based on measured visible light interferometry data.

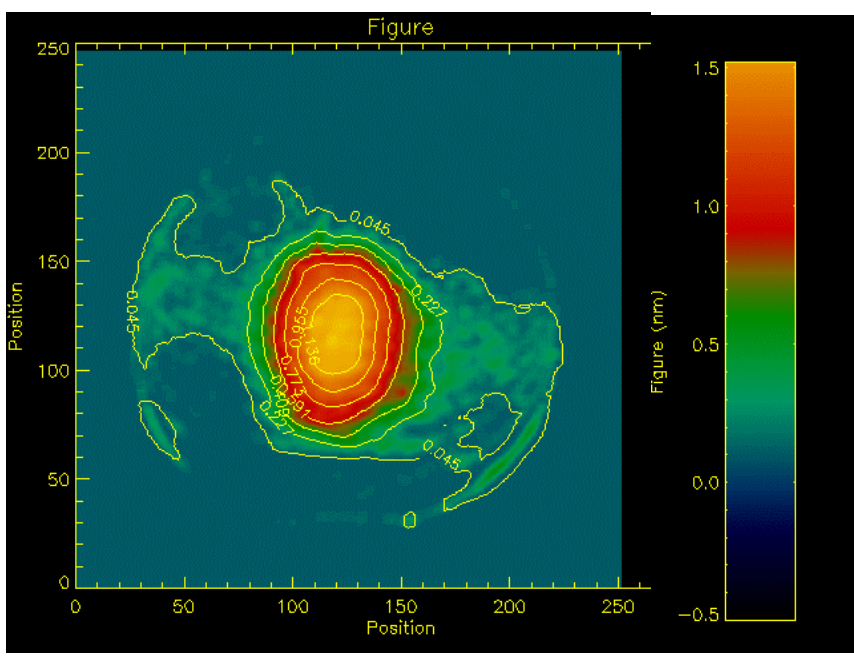

Expected figure at $13.5 \mathrm{~nm}$

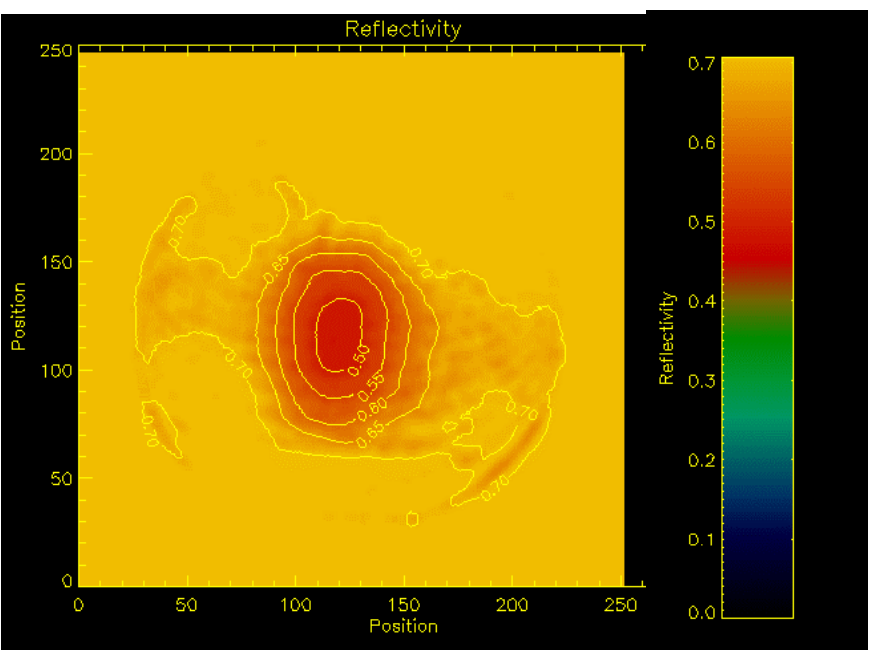

Expected reflectivity profile at $13.5 \mathrm{~nm}$

Figure 7

Expected figure (left) and reflectivity (right) at EUV wavelengths calculated from the carbon thickness map Figure 6 and the EUV figure data in Figure 6.

Note that a figure error of $1.3 \mathrm{~nm}$ and a reflectivity drop of up to $22 \%$ at EUV wavelengths is associated with the deposition of a $29 \mathrm{~nm}$ thick carbon layer. Note also that the shape of the deposited carbon closely matches that of the 10x pupil fill. Configured for small-field lithographic exposures, the 10x pupil fill is approximately Gaussian in shape, with a partial coherence of $\sigma=0.4$ in the horizontal direction and $\sigma=0.6$ in vertical direction, 
Figure 8. A comparison the calculated carbon distribution shown in Figure 6 and the pupil fill shown in Figure 8 reveal that the carbon distribution closely matches the illumination footprint on the primary mirror.

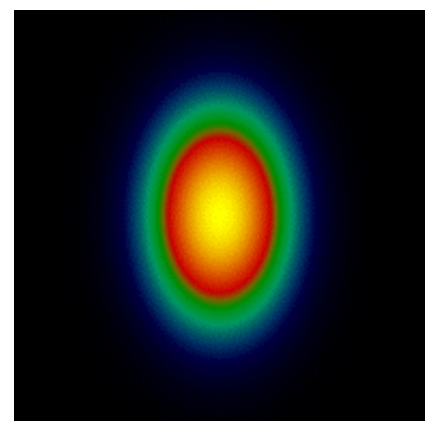

Figure 8

Approximate pupil fill of the 10x camera, which is Gaussian in shape with a partial coherence of $\sigma=0.4$ in the horizontal direction and $\sigma=0.6$ in vertical direction. Note that the shape closely matches the shape of the carbon thickness map, Figure 6.

The used optic was also inspected at EUV wavelengths at beamline 12.0 at the Advanced Light Source (ALS) in Berkeley. Although system interferometry was not performed in this case inspection of the pupil plane intensity distribution revealed a set of concentric rings that changed position as the wavelength was tuned, as shown in Figure 9. This observation is consistent with interference thickness fringes formed by interference of reflections from the multilayer mirror surface and the top layer of a Gaussian surface deposit. This observation is once again consistent with the hypothesis of a deposition on the mirror surface in the shape of the pupil fill.
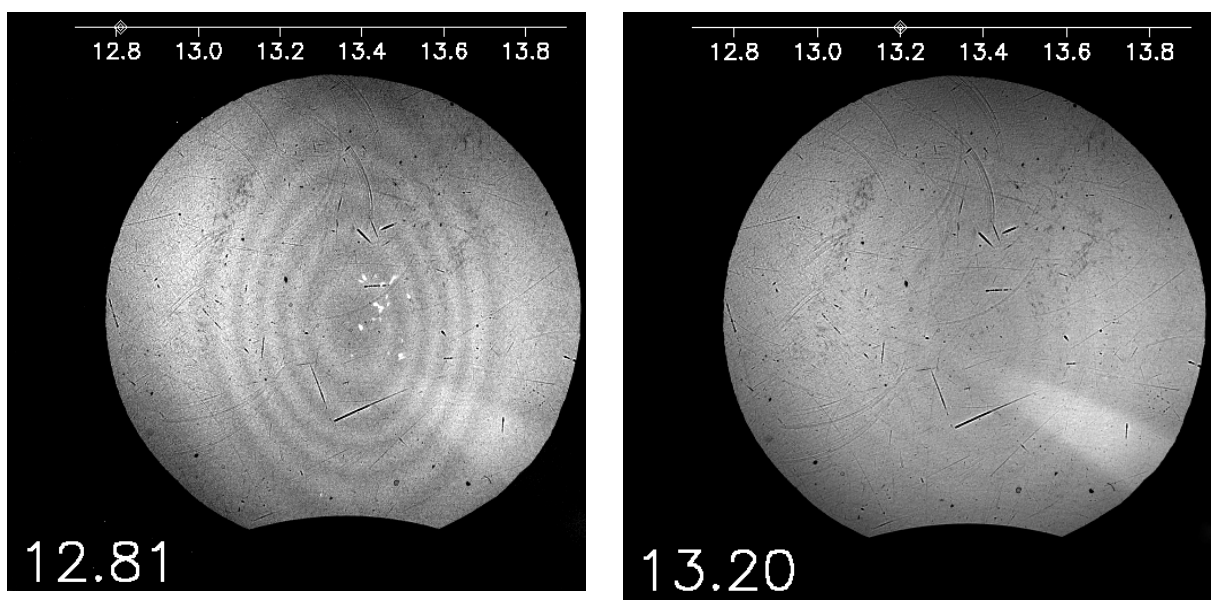

Figure 9

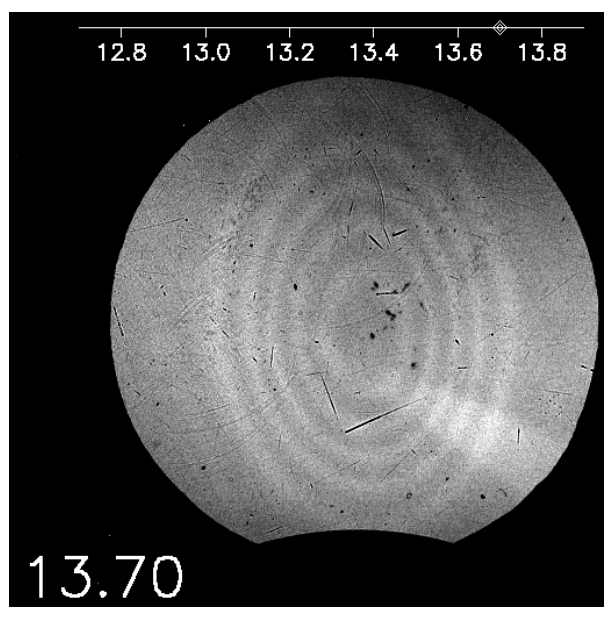

13.70

Carbon fringes observed on the 10x optics at three EUV wavelengths in the vicinity of 13.5nm

\section{Effect on aerial image performance}

To assess the effect of the observed carbon contamination on lithographic performance we performed aerial image simulations of the contaminated 10x system. These calculations were performed using a test pattern consisting of 100nm isolated and dense features as shown in Figure 10, the known Gaussian shaped 10x pupil fill of Figure 8, and using the inferred absorption and phase data shown in Figure 7 for the contaminated 10x camera. The measured figure of the 10x optic after manufacture was also known and used to model imaging in the absence 
of the observed contamination. Using a simple threshold resist model at $40 \%$ the calculated aerial images were converted into lithographic images to permit an analysis of contamination-induced changes in CD, looking at both isolated and dense features in both horizontal and vertical orientations. A collection of through-focus images with a $40 \%$ threshold resist model applied are shown in Figure 11 for the case of clean and contaminated optics, with Onm and 30nm carbon deposits respectively.

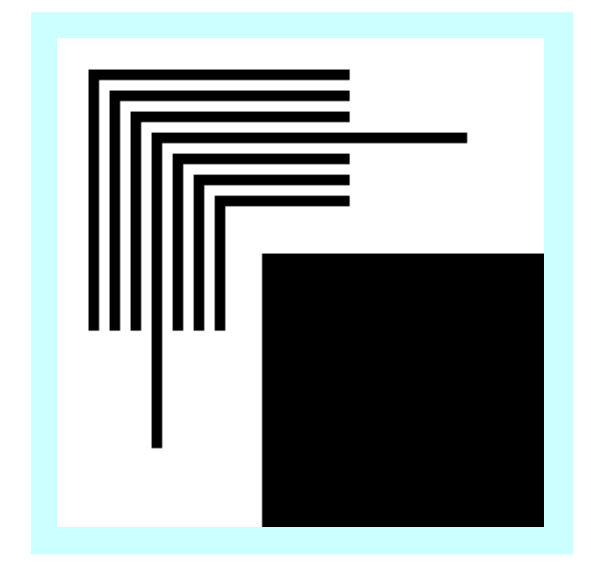

Figure 10

Nested elbow test object (left) consisting of 100nm isolated and dense features.

The effects of EUV-induced carbon deposition ion imaging performance are seen to be similar to the effects of spherical aberration, as would be expected from the shape of the carbon deposition. There is an appreciable shift in the plane of best focus and a loss of depth-of-focus with carbon deposition, whilst analysis of the original aerial images reveals a small decrease in contrast. The simulations were repeated for carbon depositions of several thickness' enabling trends in CD, contrast and defocus to be analysed as a function of contamination thickness, Figure 12. Inspection of this data shows that the most significant effect of carbon deposition is a change in focus, which can be compensated for by refocusing the tool, leaving a small change in contrast at large carbon thickness'. Interestingly there is little change in measured CD with carbon thickness. This is consistent with the observed changes in performance of this 10x camera over time, in particular a decrease in depth of focus and increase in line edge roughness (LER) observed in the printed resist image with no significant change in printed CD. 


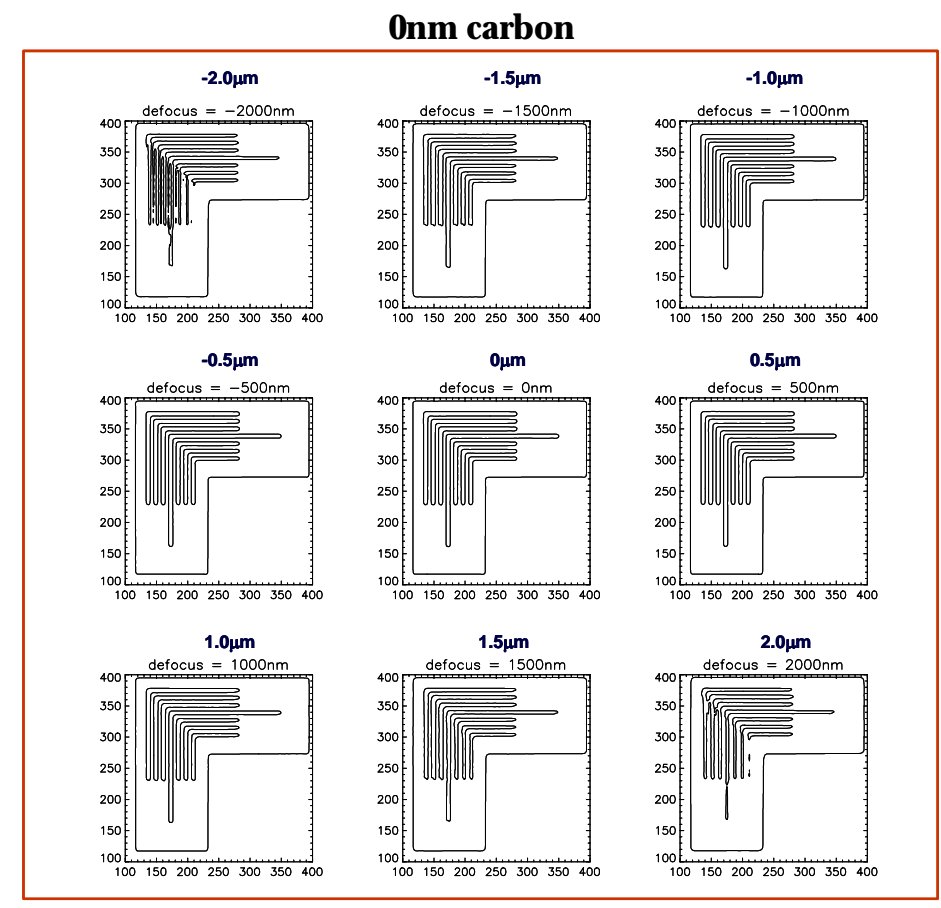

\section{0nm carbon}

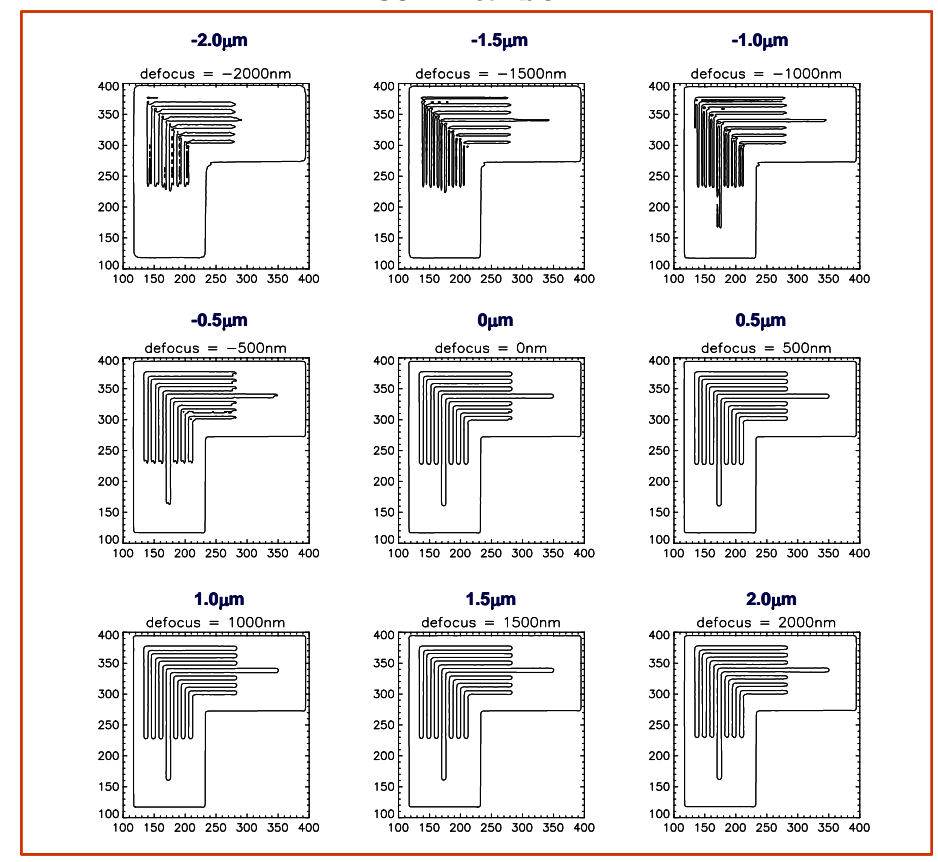

Figure 11

Aerial images through focus for a clean (top) and contaminated (bottom) 10x optic. Threshold is set at 40\%, aerial images were generated using the figure and absorption inferred from the carbon thickness map, Figure 7, and the Gaussian 10x pupil fill,

Figure 8 . 
There are two main reasons why the aerial image performance is relatively insensitive to carbon deposition. Firstly, the EUV phase change associated with carbon deposition is relatively small compared to both the carbon layer thickness and figure measured at visible wavelengths: for the case examined here a 30nm thick carbon deposit was measured as a $19 \mathrm{~nm}$ figure error using visible light, but caused only a 1.3nm figure error in the EUV. Secondly, carbon is absorbs EUV light, thus the throughput of the optic is reduced as a result of carbon deposition as expected. However the pupil is effectively apodised in the region of greatest figure error, further reducing the effect of the expected figure error on optical system performance.

\section{CD}

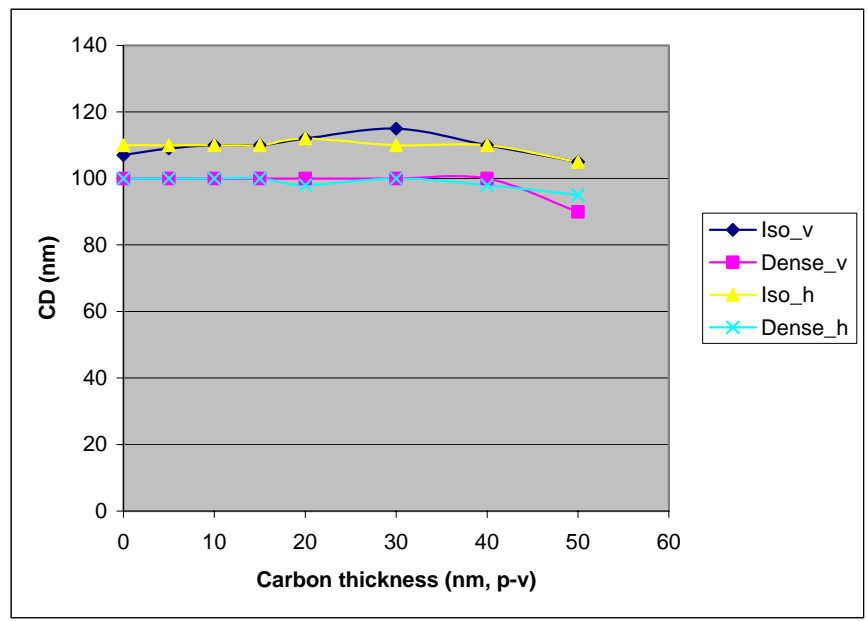

\section{Contrast}

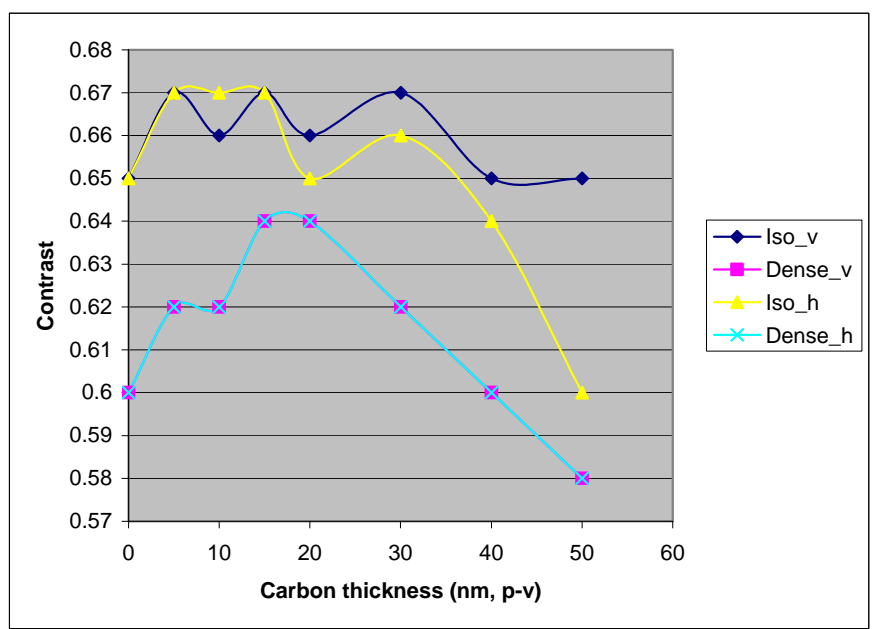

Focus

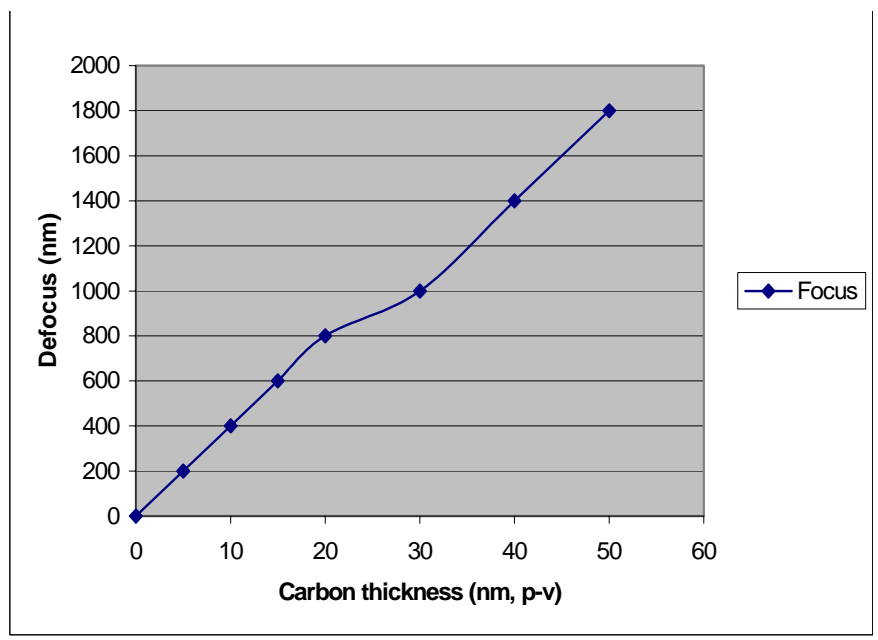

Figure 12

Variation in measured CD (top left) aerial image contrast (top right) and defocus (bottom) with varying thickness of carbon deposition. 


\section{Conclusions}

Inspection of the wavefront error of one of the Sandia 10x Schwarzschild objectives using both visible-light and EUV inspection techniques after the optic had been used in a comparatively hydrocarbon rich environment, where the hydrocarbon partial pressure is of the order of $10^{-7}$ torr, revealed a change in measured wavefront consistent with deposition of approximately $30 \mathrm{~nm}$ of carbon on the primary mirrorThis 30-nm thick carbon layer appears as a 19-nm change in measured system wavefront at visible wavelengths $(\lambda=533 \mathrm{~nm})$, however only a 1.3 $\mathrm{nm}$ figure error is observed in the EUV $(\lambda=13.5 \mathrm{~nm})$, the difference being attributable to the difference in optical constants at the two wavelengths. A reflectivity drop of up to $22 \%$ at EUV wavelengths is also associated with deposition of $29 \mathrm{~nm}$ of carbon, with the shape matching the shape of the pupil fill. The contamination reduces throughput and causes apodisation of the pupil. Configured for small-field lithographic exposures, the pupil fill was approximately Gaussian in shape, with a partial coherence of $\sigma=0.4$ in the horizontal direction and $\sigma=0.6$ in vertical direction, and the observed carbon distribution is observed to closely match the shape of the pupil fill. Significantly, the difference in predicted behaviour at visible and EUV wavelengths suggests that visible light inspection of a 'used' camera may be misleading, and that it may be advantageous to retain some form of EUV

inspection and alignment capability for use with cameras that may have some form of non-uniform contamination or deposit on the surface.

Calculations of aerial image performance for this optic based on the observed carbon deposition, taking into account both the phase and absorption of the carbon deposit as well as the pupil fill, show that the effects of EUV-induced carbon deposition are similar to the effects of spherical aberration: a shift in focus and a loss of depth-of-focus are observed in the aerial image, accompanied by a small change in contrast. This is consistent with the observed changes in performance of this 10x camera over time, in particular a decrease in depth of focus and increase in line edge roughness (LER) observed in the printed resist image.

To summarise the key conclusions from this investigation:

1. The wavefront expected to be observed at visible $(533 \mathrm{~nm})$ and EUV $(13.5 \mathrm{~nm})$ wavelengths is significantly different in the presence of carbon contamination. For the case of this optic we observed a 19nm wavefront error measured at $533 \mathrm{~nm}$, consistent with $29 \mathrm{~nm}$ carbon deposition, which in turn is consistent with $1.3 \mathrm{~nm}$ wavefront error at $13.5 \mathrm{~nm}$

2. The combination of visible light and EUV interferometry helps separate contamination from changes in figure. This suggests that EUV interferometry may be a useful tool for inspecting 'used' cameras.

3. Aerial image modeling indicates that for this particular optic the effect of carbon on imaging for this optic is reminiscent of spherical aberration. There is a change in focus and some loss in depth of focus, but only a small effect on CD. Production cameras exposed to contamination may show degraded performance reminiscient of figure errors in addition to a loss of throughput due to absorption by the carbon deposit.

This work was performed under the auspices of the US Department of Energy by the University of California, Lawrence Livermore National Laboratory under contract No. W-7405-Eng-48. Funding was provided by the Extreme Ultraviolet Limited Liability Company (EUV LLC) under a Cooperative Research and Development Agreement. UCRL-JC- 
University of California

Lawrence Livermore National Laboratory

Technical Information Department

Livermore, CA 94551

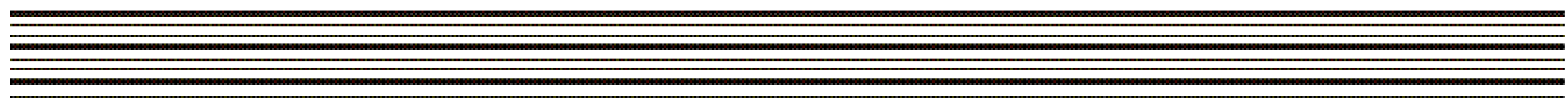

